\title{
Development and Evaluation of a Software-based Clinical Pharmacography System
}

\author{
Nicolae TODOR ${ }^{1}$, Mihai BORZAN ${ }^{2 *}$, Laura Elisabeta CIULEANU ${ }^{3}$, Dana Ioana IANCU ${ }^{4}$, \\ Voichita Alexandra GHENGHEA ${ }^{5}$, Tudor Eliade CIULEANU ${ }^{4,6}$ \\ ${ }^{1}$ Biostatistics and Medical Informatics Department, \\ "Prof. Dr. Ion Chiricuta" Oncology Institute, \\ 34-36 Gheorghe Bilaşcu Street, Cluj Napoca, 400015, ROMANIA \\ todor@iocn.ro \\ ${ }^{2}$ University of Agricultural Sciences and Veterinary Medicine, \\ 3-5 Calea Mănăştur, Cluj Napoca, 400372, ROMANIA \\ mihai_borzan@yahoo.com, ${ }^{*}$ Corresponding author \\ ${ }^{3}$ SC Vetomed SRL, \\ 171 Calea Turzii, Cluj Napoca, 400495, ROMANIA \\ lizi_cluj@yahoo.com \\ ${ }^{4}$ Oncology Department, Oncology Institute „Prof. Dr. Ion Chiricuta”, \\ 34-36 Gheorghe Bilaşcu Street, Cluj Napoca, 400015, ROMANIA \\ dana_iancu2004@yahoo.com \\ ${ }^{5}$ Communications in Foreign Languages Department, \\ "Politehnica" University of Bucharest, \\ 313 Splaiul Independenței, Bucharest, 77206, ROMANIA \\ vghenghea@gmail.com \\ ${ }^{6}$ Oncology Department, "Iuliu Hatieganu" University of Medicine and Pharmacy, \\ 34-36Victor Babeş Street, Cluj Napoca, 400012, ROMANIA \\ e-mail: tudor@iocn.ro
}

\begin{abstract}
Objectives: Prescribing, dosing and delivering chemotherapy to the cancer patients is a complex, highly specialized and time consuming process, requiring a specialized team (medical oncology clinician or veterinarian, nurse, pharmacist). Our aim was to create a software that fills the gap between medical doctors, pharmacists and nurses and consequently speeds up the calculation, dosing and preparation of chemotherapy and biological (targeted) therapy in medical oncology. Dedicated versions for human and veterinary medicine are available.

Results: First version (chemotherapy and hormonal therapy in humans) was created in 1997 and the software was continuously updated. The 2014 update includes four versions (i.e. for humans or pets and standalone or network). The protocol library was extended to more than 800 chemotherapy protocols to include new chapters such as molecular targeted therapy and immune oncology. All 50 requirements and standards for achieving a good prescription from EuroRec EHR Quality Seal Level 2 are fulfilled. In our institution (Oncology Institute Prof. Dr. Ion Chiricuta) more than 300000 chemotherapy cycles were already administered using this software. The program is also used in eight county hospitals or private centres in Romania. A customized version was recently created for use in the veterinary medicine (cats and dogs).

Conclusions: The presented solution, a robust software program makes the administration of chemotherapy safer, avoiding calculation errors, offering instant access to patient's history, as well as to a vast and updated protocol library. It shortens the time, standardizes the process and reduces the workload related to chemotherapy. The software can provide to the health authorities real-time data related to the number of cancer patients treated, the indications and the amount of drugs used. Its veterinary version can help the development of a veterinary medical oncology network. The data from this software may be used for prediction of stocks in pharmacy. The chemotherapy protocol library with browsing and help system attached provides a basis for an e-learning system in oncology.
\end{abstract}

Keywords: Chemotherapy software, human chemotherapy, veterinary chemotherapy.

\section{Introduction}

Medical oncology is one of the most dynamic areas of contemporary medicine. In addition to standard chemotherapy and hormonal therapy, medical oncology currently includes molecular targeted therapy (monoclonal antibodies, protein kinase and immune checkpoint inhibitors) and supportive care (erythropoiesis stimulating agents, growth factors, osteoclast inhibitors, antiemetics, analgesics). The large set of products and possible combinations require a computerised infrastructure for individual, institutional, regional or national use. Some of the pharmaceutical companies provide for their specific products nomograms or developed 
programs that help the physician in using these molecules to avoid toxicity. Various patents offer different prescribing solutions [1], such as: JP2004362336(A) "Cancer chemotherapy execution support system" JP2007293906(A) "Cancer chemotherapy management system", US2006/0031100AI "Method of providing per dose delivery of veterinary oncology chemotherapy and immunotherapy agents and nutritional formulations". Some leading companies or cancer centers have created their own utilities such as ARIA product by VARIAN or the IntrinsiQ program, of Miami University [1]. Since 2007, the software application, IntelliDose, permitted the registration of 17000 patients each month. This data base is the foundation of products and services with multiple beneficiaries: it facilitates the activity of the treating physician (oncologist) and clinical pharmacist, eliminating the human error in chemotherapy dosing and scheduling, facilitates the decisions of the hospital manager, and is useful to the market researcher and the financial analyst. Despite a major request, there are few global solutions developed, even for human oncology, and almost completely lacking in veterinary oncology.

In Romania, the prescription of chemotherapy is still done manually, without using a computerised system, and the chemotherapy doses are individually calculated by the oncology physicians, for each patient, using published chemotherapy protocols and nomograms for body surface calculation. This process is time consuming and leaves the space for human error. The electronic prescription recently introduced in Romania by the health authorities does not cover the complexity of chemotherapy protocols calculation in an individual patient.

In order to achieve high quality standards for the medical oncology department, the Oncology Institute "Prof. Dr. Ion Chiricuță" Cluj-Napoca (IOCN) created, since 1997, a pharmacography software for medical oncology, that was periodically updated, with the last update in 2014 [1]. The protocol library was extended to include new chapters such as molecular targeted therapy and immune oncology.

At present, the database includes 800 chemotherapy protocols and over 300,000 cycles administered to patients between 1997 to 2015. Because of its practical advantages and friendly use, the solution is currently implemented in other cancer services in Cluj (Department of Radiology of the Medical County Hospital and Medisprof Clinic) and in some other county cancer cent (Alba-Iulia, BaiaMare, Deva, Oradea, Sibiu, Târgu-Mureş,).

Recently, the program was adapted also for use in veterinary medicine, a unique solution for Romania until now. The veterinary knowledge data base includes 50 protocols for the chemotherapy treatment in pets (dogs and cats).

The 2014 update includes four versions (i.e. for humans or pets and standalone or network).

\section{General Overview of Chemotherapy Treatments and Derived Requests}

Because the original version was designed for human medicine, we shall refer to this version and the differences will be indicated where appropriate for veterinary medicine.

First we present the physician point of view with minimal references to information technology.

At each visit the prescribing physician in dialogue with the patient fulfils the following steps: evaluation, input data analysis, recommendations, selection and adaptation of the chemotherapy protocol from panel options. The process is continued with the preparation of the treatment (by the pharmacist, nurse or physician) and the treatment administration (by the nurse or the physician).

Evaluation involves the study of the patient's documents together with a clinical examination and additional analysis, if needed.

Input data analysis is a step where from the total set of information concerning the patient, the physician selects the relevant data.

Recommendations, is the step in which the treating physician has access to all the information related to the chemotherapy protocols and guidelines, together with the legal regulations and eventual restrictions. In this step obviously the computer is needed. The medical doctor (prescriber) browses electronically the protocols with the aim of choosing the optimal one.

Selection $\&$ adaptation from panel options is an important step where the physician validates the optimal decision for the patient. No need to stress the importance of computer by which 
simulations or other auxiliary estimations can be done. The final issue is prescribing chemotherapy for the pharmacy department.

Pharmacist's preparation phase implies the preparation of the infusion respecting the prescription received. This step in a way is a specific black box but the main computer facility is referring communications between physician and pharmacist.

Treatment administration is the final step fulfilled by physician's team. In this step it is important to notice (also in the database) any impairment (modified schedule, allergies) which could be useful at next visits.

Figure 1 presents a flowchart with all these activities. The key problem for physician and further for computer scientists is how to navigate between the database with personal data of patients and the knowledge chemotherapy database.

The core of the program links patient's specific data (personal data, diagnosis, age, weight, height, treatment history) with appropriate treatment regimens for generic diseases resulting a customized treatment plan for the patient. All various possible equivalent options are suggested to the physician and he/she will decide in this context on the future treatment of the patient.

Concerning knowledge chemotherapy database, another intrinsic part of the program, the computer program has to offer the possibility of a simple and continuous updating of the regimens and schedules of administration, so dynamic in modern oncology.

In the last version of the software, the protocol library abbreviation for each cytotoxic agent was unique, respected an alphabetic order and was limited to three characters. The library we proposed represents a novelty among a very heterogeneous and confusing denomination of the cytotoxic agents in the literature. For instance cisplatin is abbreviated as $\mathrm{C}, \mathrm{P}, \mathrm{Pt}$, DDP or CIS in different regimens, while in our library it is always represented as CIS.

Medical practice requires close monitoring of the patient during chemotherapy administration including notification of events which occur and may affect the future evolution of the patient. To prevent the creation of two parallel systems the production of any document outside the database connection has been banned. Corrections are not allowed if done by hand. A document to be corrected is first cancelled, then a correction is generated into a new document in connection with databases.

Knowledge database is modified only with the approval of hospitals' special committees of treatment, including different oncology specialists.

Starting from this, an ad hoc code of 'software good practice' in four points was developed:

$\mathrm{C} 1$. All documents including corrections must be generated through the computer in connection with the system of databases, thus ensuring maximum coherence of subsequent processing in the databases. In short terms that means that handwritten documents are forbidden.

C2. Remove the physical delete function on databases and replace it with a "cancellation" by attaching to each item in databases at least three states: editing, validation and cancellation. In common exploit of databases only validated items are used.

C3. The default displays historic items from any database, including articles that are cancelled. Special markings for easy viewing

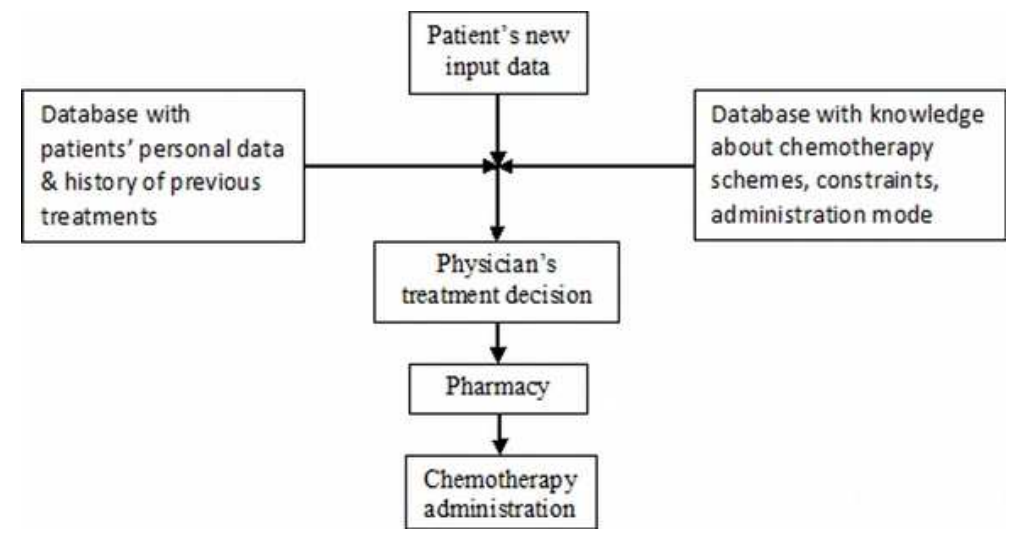

Figure 1. Activities implied in each visit for chemothrapy administration 
by the human operator are requested. In the last version in all databases cancelled items are marked by the sign $\mathrm{X}$ displayed in red and in the background of the screen; for editable items the same procedure is used as for cancelled items but the sign $\mathrm{X}$ is replaced by $\mathrm{a}$; validated items are free from any sign.

C4. Availability to exchange information with other systems based on the special structure of separators messages like HL7 system [2].

All software modules developed in / for IOCN comply with this requests. Another example on the information system covering radiotherapy within IOCN respecting $\mathrm{C} 1-\mathrm{C} 3$ is detailed in a previous publication [3].

A system that is intended as a practical tool for the medical oncologist or veterinarian is further necessary to have a fast and coherent connection to the pharmacy implied in the preparation of cytotoxic drugs. Also counting on the dynamic change of new available drugs, the system needs a performing maintenance mechanism for an easy update of the knowledge database.

The actors in the current activity of chemotherapy administration to cancer patients are doctors, pharmacists and nurses. They must be identified in the computer system and receive differentiated user access rights to information. Of course we need a special user called "administrator" which is designed to feed the knowledge database with new treatment regimens and other system parameters as the information about all users. The administrator must be the interface between the system and the authorities, government, house of insurance and so on.
The physician is the most dynamic actor, having major rights throughout any treatment. He may cancel or change any information in the system, still subject to the conditions $\mathrm{C} 1-\mathrm{C} 3$ which are keeping tracks of changes and cancellations. The most important activity of the physician and of the entire system is the prescribing of the chemotherapy protocol adapted to the individual patient characteristics. According to some authors $50 \%$ of chemotherapy errors occur here [4] and that is the reason that we assign a large space to this.

\section{Software Description}

The operating diagram of the software, types of users and write accesses are shown in Figure 2. The backbone of the program consists of nine databases: Users, Prescriptions, Knowledge sets, International Classification of Diseases for Oncology (ICDO) codes, Histopathology (HP) codes, Nurses, Residents, Insurance (Ins.) Houses, Trials.

1. Users is a database which is automatically accessed by the program at each entry or exit from a user's system or at every new user request. The administrator is the only user who has rights to initiate changes or to generate new articles in this database.

2. Prescriptions is a database where all the details relating to the identification, management and deployment of chemotherapy administration to patients will be deposited. Generating swift and accurate articles in this database is the main purpose of the software.

3. Knowledge sets form the database containing generic schemes with drug regimens and health-related constraints.

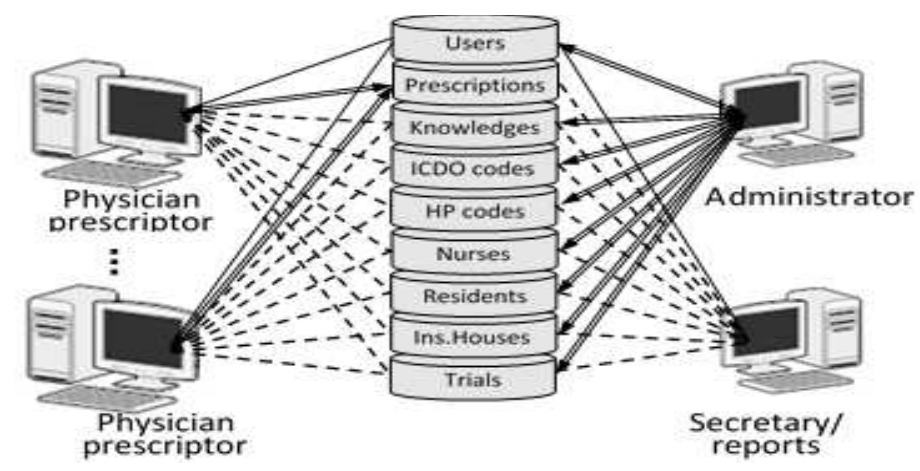

Figure 2. Functional scheme and databases accesses

$(\mathrm{ICDO}=$ International Classification of Diseases for Oncology; $\mathrm{HP}=$ histopathology; double arrowed line $=$ read - write access; single line $=$ read access; dotted line = read access and partial write access) 
During the prescribing process, an item filled only with generic medical information is generated from this database. In this item the physician sets the actual status of the patient right before the start of chemotherapy administration (such as weight, serum creatinine) and which is the selected scheme to be prescribed.

4. ICDO codes is a database related to the malignant registry model of World Health Organization [5]. It is used to produce a uniform exchange of information with institutional or regional malignant registries and pathology services.

5. HP codes is also a database related to the malignant registry model of World Health Organization [5]. It is used to produce a uniform exchange of information with pathology services.

6. Nurses is the database updated by the administrator that contains the list of all nurses involved in the administration of chemotherapy. The physician mandatory assigns a nurse for each patient when prescribing chemotherapy.

7. Residents is the database updated by the administrator containing the list of residents involved in prescribing chemotherapy.

8. Insurance Houses is a database containing mainly the list of insurance houses by which the administration has agreements. At the start of prescription the physician has to select the right option for each patient in order to obtain the reimbursement.

9. Trials is the database containing the catalogue with the clinical trials available. It is updated by the administrator with information about trials approved by the institution. Could be an empty list.

To better understand how the system works we'll make some remarks that apply to any software implemented in IOCN no matter the area:

1. The symbol consisting from multiple arrows to the left placed in proximity of a field (i.e. NID), in the next figures enables us to bring-in external information that is integrated with the information already on the screen. The most common use refers to taking the patient's personal data or information about malignant tumour registry based on number of identification of the patient.

2. The symbols consisting from single arrow up or down with optional horizontal mark refer to the navigation through active data collection. They involve a jump to the first item of the collection; go to the previous article; go to the next; go to the last item. Buttons engraved with "Omit" skip the current item in the collection; engraved with "Show omitted" find complementary items collection; engraved with "Show all" implies the expansion of active collection to the entire database. In the proximity of these buttons are always shown the total number of items of the database, the total number of items and order number of the item in the current collection. Clicking the area that contains this number actually the computer asks for access to an item whose serial number should be indicated by typing.

3. Most important menus showing information from databases always contain an area marked with the words Create, Validate, Cancel, Print. In the fields located in the proximity of these areas is stored all the information about the user that caused the creation, validation or print of that item. The index of user, name and surname, title, type, function and user department followed by the date and time when the event occurred are all stored. This information is connected with action buttons engraved with New, Validate, Cancel, Duplicate and Print.

4. We must not forget activities of information search in databases. It allows the operator to position on the item or collection of items that will be modified, validated, printed or cancelled. Basically a search in a database supposes a selection after one or more filters. Practically a filter is a blank image of a database where the user completes the information on which items must be selected. Search specific functions are associated with buttons engraved with the words Find, New Filter, Negate Filter, Execute Find, Execute extended Find, Execute constrained Find, Cancel Find. A search is launched by pressing the button engraved by Find or Modify Find. For the first option it is understood that all filters are built from scratch. Modify Find instead take the filters used in the last search but the 
operator can add new ones, can browse, edit, negate or delete. Finishing or the completion of a search is done by operating one of the buttons engraved with Execute Find, Execute extended Find, Execute constrained Find or Cancel Find. Execute Find and Cancel Find does not require further explanation. Execute extended Find assumes that the search result is added to the active collection items. Execute constrained Find involves that the request is performed on the active collection. In addition, during search we have available a series of navigation functions through filters that have the same symbolism and meaning as at point 2 above.

From the nine databases shown in Figure 2 we present only the most important: the database of knowledge and database of prescriptions.

Figure 3 is an example of a real prescription of a chemotherapy protocol, called TIP (CIS + IFO + PTX, meaning cisplatin + ifosfamide + paclitaxel) used in patients with a relapsed germ cell tumour. For a better understanding we first pass over the administrative part and we focus on the medical side:

1. One part of observations (OBS) evidencing general things about the scheme i.e.: premedication, restrictions, toxicity to follow during administration. This information is text free and the user can store there specific treatment aspects of the clinic.

2. The long name of the scheme (Long Name) which is intended to be the full name of the scheme without abbreviations. The database currently used to IOCN including the example has been filled usually by cytotoxic drugs name if there is no other version dedicated.

3. Recommendations (the middle part) must indicate the tumour sites where the protocol is used, as recommended by the clinical guidelines. Correct completion turns into an important help to the prescriber. In the figure we see two recommendations validated (ovary and testis), a cancelled recommendation for mesothelioma and a recommendation which is in editing

4. The references were added to indicate the sources used in documentation and in thisway to fix the system boundaries.

$\mathbf{Z 1}$ : the day of onset of the cytotoxic drug. It is compulsory for each line.

Z2: the last day of the administration of the cytotoxic agent. Missing if there is only one day of administration in which case only $\mathrm{Z} 1$ is filled.

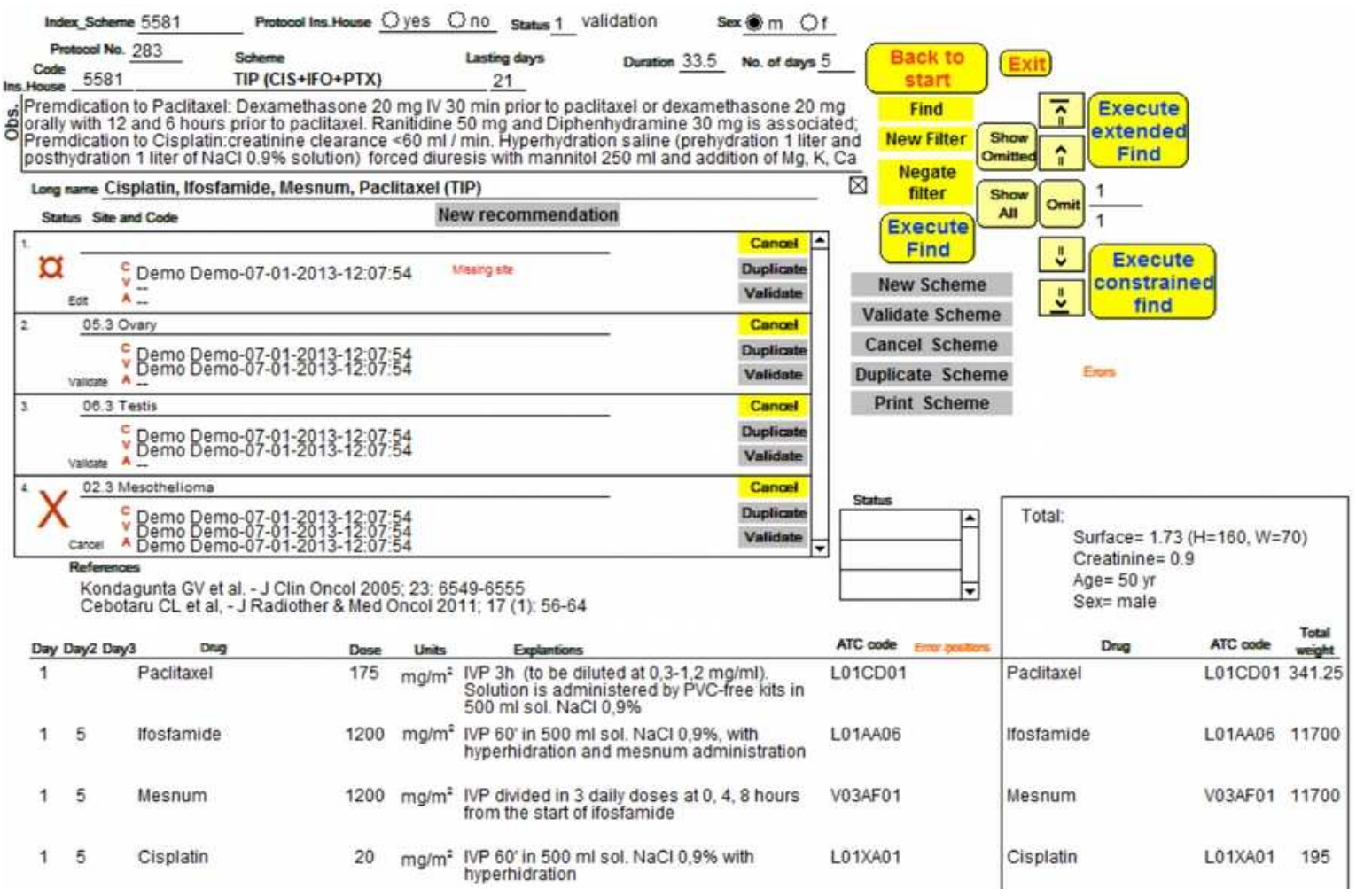

Figure 3. Creation and update example of a protocol of treatment in knowledge database 
Z3: the periodicity of administration within a treatment cycle. It is present only where appropriate.

Cytostatic column must be completed and contains the name of the active drug to be administered.

Dose columns and Units of measure are relatively clear due to their suggestive names. Possible units of measure are $\mathrm{mg} / \mathrm{m}^{2}, \mu \mathrm{g} / \mathrm{m}^{2}$, $\mathrm{MU} / \mathrm{m}^{2}, \mathrm{U} / \mathrm{m}^{2}, \mathrm{mg} / \mathrm{kg}, \mu \mathrm{g} / \mathrm{kg}, \mathrm{MU} / \mathrm{kg}, \mathrm{U} / \mathrm{kg}, \mathrm{mg}$ DT, $\mu$ g DT, MU DT, U DT, AUC, where $\mathrm{U}=$ units, $\mathrm{MU}=$ million units, $\mathrm{DT}=$ total dose, $\mathrm{AUC}=$ area under curve).

Column ATC code ("anatomical, therapeutical and chemical code") was introduced to ensure full interoperability with scientific names required by the authorities.

As a corollary from medical part, the administrative part consists of several fragments which are also marked in bold for easy identification. These are the following:

1. Index_Scheme: denotes the index scheme or protocol that will be used internally by computer.

2. Protocol Ins. House: indicates whether or not the scheme is paid by the insurance company.

3. Status: has possible values discussed above: editing, validation and cancellation.

4. Sex: $m$ or $f$ are possible values for male and female completed only if the scheme relates to a protocol that is assigned exclusively in function of patient's gender.
5. Protocol no.: indicates the number of protocol as used in reporting.

6. Code Ins.House: is the insurance house code and is used for reporting in this direction.

7. Scheme: is the code scheme as used ineveryday language of those who prescribe and administer chemotherapy (ex. BEP, EP, TIP, CHOP, etc.).

8. Lasting days: indicates how many days remain before the start of following administration.

9. Duration: is the number of hours required for administration. It is used to assign the complexity of the treatment for the insurance house.

10. No. of Days: the number of days in which the patient needs care. As Duration at point 9 this information contributes to the evaluation of the complexity of the scheme.

In addition to the ten points above the right side of the screen contains an evaluation of the necessary chemotherapy for a mean patient for administrative purposes.

In Figure 4 we have the command menu used in building prescriptions.

The resemblance to the knowledge database is natural. Link to Knowledge Base is made through treatment protocol code that is "BEV + CBP + PTX bp" in the selected example (meaning bevacizumab + carboplatin + paclitaxel for bronchopulmonary cancers). When the code

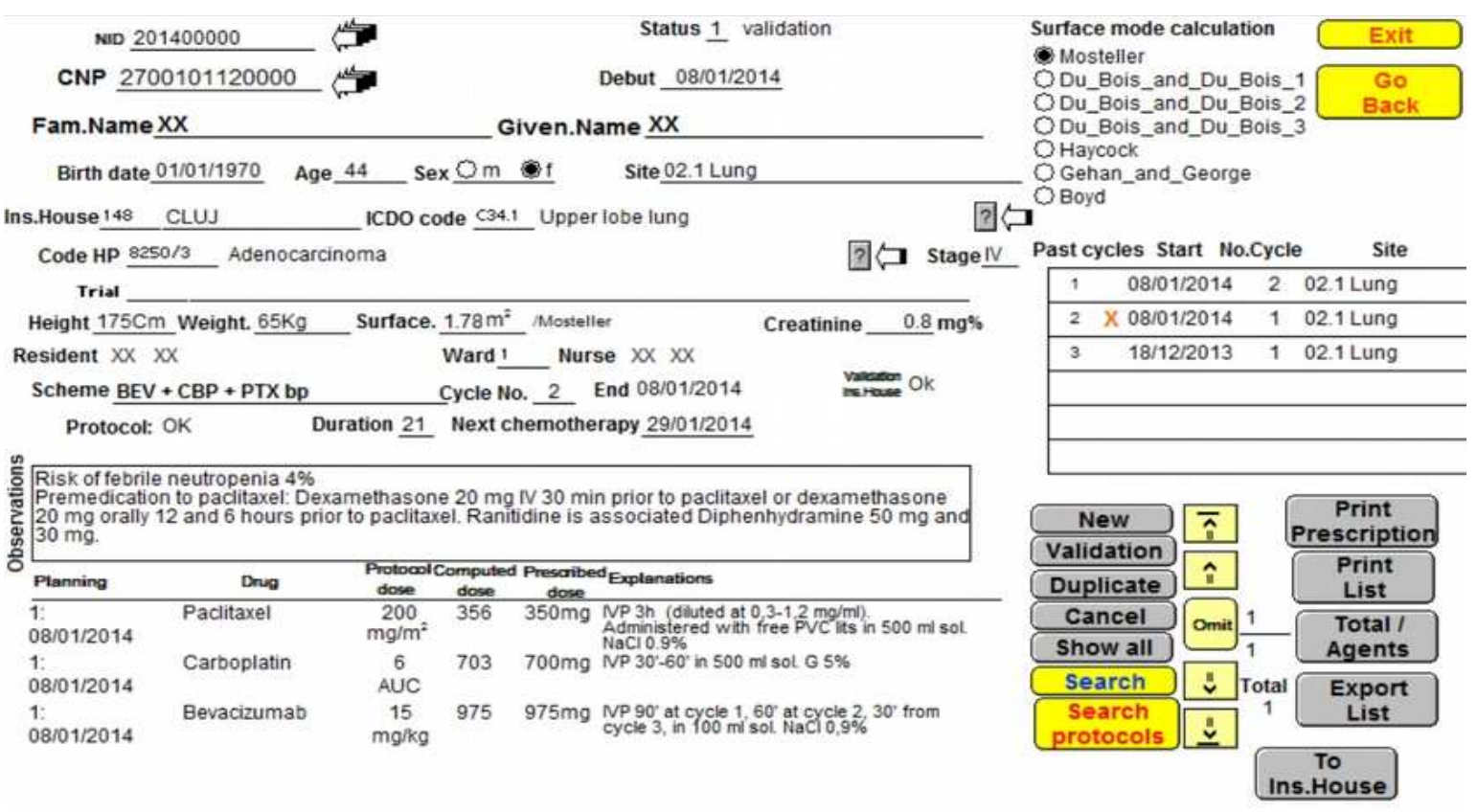

Figure 4. Example of prescribing a chemotherapy protocol 
scheme is completed, the knowledge database is loaded entirely with the appropriate description of treatment. This description is completed with information about the patient: sex, age, height, weight, and serum creatinine, and leads to the calculation of the theoretical dose for the patient. The prescriber may adjust this dose if necessary, validate the schema, then print and launch the preparation and administration of chemotherapy.

In chronological order the prescriber as in Figure 4 first loads administrative data: internal identification number, identification number for the house of insurance, name and surname, current date, gender, resident, nurse, trial, cycle number, room number, followed by medical information: tumour site, ICDO code, histology code, stage, height, weight, chosen treatment, calculation option for body surface area.

Further we underline two functional aspects:

1. Site of the primary tumour should becompleted at the beginning so that suggestions can be made further according to the knowledge base. Chapters of tumour site comply with classic chemotherapy guidelines $[6,7,8,9]$.

2. The calculation of the dose in chemotherapy is the most often related to the body surface. This is better correlated with the cardiac debit and the liver or renal clearance of different cytotoxic agents than the body weight. However, different modalities of calculation apply for different agents, and these are known from the chemotherapy protocols published in the literature for various tumour types. The modality of calculation for the different protocols is described in the knowledge database. When we prescribe per kilogram $(\mathrm{mg} / \mathrm{kg}, \mu \mathrm{g} / \mathrm{kg}, \mathrm{MU} / \mathrm{kg}, \mathrm{U} / \mathrm{kg})$ things are simple, take the patient's weight and multiply by the corresponding dose. The simpler is the case when administered cytostatic is for a total dose (mg DT, $\mu \mathrm{g}$ DT, MU DT, U DT) in which case there is no need to multiply. Complications arise when the administration is following body surface area $\left(\mathrm{mg} / \mathrm{m}^{2}, \mu \mathrm{g} / \mathrm{m}^{2}, \mathrm{MU} / \mathrm{m}^{2}, \mathrm{U} / \mathrm{m}^{2}\right)$ or area under the curve (AUC) related to clearance of creatinine. The reason is that both dosage forms calculation are not based on direct measurements, but are estimate functions depending on weight (W) and height $(\mathrm{H})$.
For body surface area (BSA) we have integrated five estimators in humans:

- Du Bois 1916: $0.007184 \times \mathrm{W}^{0.425} \mathrm{xH}^{0.725}$ [10]

- Gehan 1970: 0.0235W $\mathrm{W}^{0.52456} \mathrm{xH}^{0.42246}$ [11]

- Haycock 1978: 0.024265 $\mathrm{xW}^{0.5378} \mathrm{xH}^{0.3964}[12]$

- Mosteller 1987: (WxH) $)^{0.5} / 60$ [13]

- Boyd 1998:0.03330 $\mathrm{W}^{(0.6157-0.0188 \log 10(\mathrm{~W}))} \mathrm{xH}^{0.3}[14]$

The formulas are similar but can induce notable differences. Thus for an average patient in Romania with $\mathrm{H}=160 \mathrm{~cm} \mathrm{~W}=70 \mathrm{~kg}$ and having a body mass index (BMI) of 27.3, calculating BSA for all possibilities we get a difference between the maximum and minimum of $697.3 \mathrm{~cm}^{2}$ ie $4 \%$ comparing with minimum. For an underweight patient with $\mathrm{H}=$ $168 \mathrm{~cm}, \mathrm{~W}=54 \mathrm{~kg}$ and $\mathrm{BMI}=19.1$ the value of the difference is only $2 \%$. Although apparently numerical differences are small i.e. below $5 \%$, these differences may be important in terms of health and therefore the authors recommend using the same formulas as that used in the chemotherapy study launching the chemotherapy agent. If such information is not available the recommendation is to use the formula of DuBois [10] which was first recognized for this goal, even in spite of the simpler and popular Mosteller formula [13].

In figure 4 formula for BSA is chosen by selecting the appropriate option in "Surface Mode Calculation" zone on the up-right.

In dogs, the formula commonly used is BSA $\left(\mathrm{m}^{2}\right)=10 \times \mathrm{W}^{2 / 3}$ where $\mathrm{W}$ is the body weight in grams. For small dogs (less than $10 \mathrm{~kg}$ ) and cats, the validity of dosing chemotherapeutic drugs based on BSA was questioned with regard to increased toxicity for certain drugs (doxorubicin, cisplatin, carboplatin and melphalan). The current recommendation is to use body weight $(\mathrm{mg} / \mathrm{kg})$ to dose certain drugs (e.g. doxorubicin) in dogs that weigh less than $15 \mathrm{~kg}$ and in cats.

For AUC used exclusively in administration of carboplatin we use the Calvert's formula [15]. Although there are discussions about corrections that should be applied [i.e. 16, 17] we have for now left to the discretion of the physician the option of manually modifying the dose where necessary.

Calvert's formula evaluates first glomerular filtration rate (GFR) with (140 - age) $\mathrm{x} \mathrm{W/}$ (serum creatinine $x$ 72) +25 for men and 
decreased with $15 \%$ for women. This value multiplied by AUC from knowledge database is proposed as total dose of carboplatin.

\section{Discussions}

Quitting handwritten diagrams clearly increased clarity of communication between all players involved in chemotherapy treatments without counting the speed of development of prescription.

Implementation of a commercial computer system for prescribing chemotherapy is a complicated task. For one of the most successful system at Tisch Cancer Institute, New York the total duration of implementation took over two years [23]. Being a "top-down" implementation we have to add, together with the specific activities involved, a series of computer training modules, analysis with monthly meetings throughout the implementation.

In contrast we had "down-top" realisation. It began rather like a play, without formal employment. The requirement was rather ambiguous: "Could computer solve the numerous repetitive laborious operations, associated with the administration of chemotherapy?" The response was positive and thus was born the version 0 of the program. The knowledge database needed to be expanded to the whole pathology of IOCN and from 1997 the administration decided as mandatory the use of electronic prescribing.

IOCN is a regional centre for training, and former residents dispersed across the region and wanted to use the chemotherapy software in their own institutions.

Only the part that refers to prescribing chemotherapy was presented above. Implementation in IOCN is done on a larger scale including pharmacy, nurses and staff who usually do not administer chemotherapy (surgeons and radiation oncologists).

The chemotherapist initiates the treatment by generating a record in the database of prescriptions, then, in sequence the pharmacist and nurse take the hand. Nurses and pharmacists have read only access in general to whole system and full access to their specific area which have to be filled in order that the treatment starts.

Only chemotherapist (prescriber) can cancel and/or generate another record.
The surgeon and radiotherapist and other categories of staff implied in treatment have read only access.

Special timing rules are implemented. For example nurses can't modify before pharmacist validation of the record or pharmacist can't do the dilution of drugs before medical doctor's validation.

When the relatively simplistic original version of the program was launched in 1997 there were only few solutions to make a reasonable choice of possible software options. New challenges have occurred due to changes in computers facilities and dynamic changes in chemotherapy protocols. So if at the beginning there were only a few dozens of protocols, currently we have 800 protocols including not only solid tumours, but also haematology, paediatrics and veterinary medicine. Now the total number of annual prescriptions using the program is over 33,000 only in IOCN.

Worldwide there are many commercial solutions. American Society of Clinical Oncology (ASCO) makes a series of recommendations based on requests of vendors to ASCO for presentations of products at ASCO meetings [24].

Our current information system is developed based on the management system of databases FileMaker6 which in server version admits maximum 254 users. The system fulfils all 50 requirements from "EuroRec EHR Quality Seal Level 2" [18]. This allows connections with other systems of IOCN developed in FileMaker or other management systems databases. When prescription system is used in a different context from the requirements cited above only the following five could pose problems or need further attention:

1. Request 4 [18]: The system displays all current health problems associated with a patient.

2. Request 10 [18]: The system presents a medication history associated with a patient.

3. Request 14 [18]: The system presents a list of the allergens with an active status.

4. Request 21 [18]: The system is able to present a list of the individual results for a discrete lab test for an individual patient.

5. Request 34 [18]: All patient data can be accessed directly from the patient record. 
In Figure 5 we have the "canine" version of the program. It is observed the similarity with human version. However there are some

differences in both the administrative and the medical side. Thus we are obliged to complete information about the owner and race and relationship with insurance house disappears.

Canine body surface area can be calculated from the formula of Lowe [19] $\left(10 \mathrm{xW}^{2 / 3}\right.$, where $\mathrm{W}$ is the weight expressed in grams), or may be extrapolated from the tables of Löscher [20]. Unlike humans calculation of body surface height has no role. For dogs with $\mathrm{W}<10 \mathrm{~kg}$, the dose is preferably related to the weight calculation, which produces fewer side effects, especially for some cytostatics such as doxorubicin [22].

Compared with human complex pathology, canine pathology in the database is reduced to 12 categories: carcinoma, transitional cell carcinoma, sarcoma, osteosarcoma, mast cell tumours, central nervous system, lymphoma, leukaemia, myeloma, myeloproliferative syndrome, thrombocytopenia and other tumours. The knowledge database of this version of chemotherapy used as support schemes presented in the volume of Withrow and MacEven's [21]. Unfortunately there are no national or European accepted therapeutic guidelines for veterinary oncology.

For humans, interested people can download from http://srrom.ro/Adela/Demo_Prescription_
Install_en.pdf a free demo version of the program. Each downloaded copy is a complete standalone program but with no network capabilities and limited at 300 prescriptions i.e. 50 patients at a mean rate of 6 schemes per patient.

\section{Conclusions}

Chemotherapy prescribing software created at the Institute of Oncology "Prof. Dr. Ion Chiricuta" Cluj-Napoca proved to be one of the most robust software programs used in Romania so far. Outside IOCN it is used with minimal adaptations in eight county hospitals or private centres.

The program is fully compliant with "EuroRec EHR Quality Seal Level 2"and only minimal adaptations are required for compatibility with the other local administrative systems.

The benefits of immediate use are linked to increased quality of cancer care in human and veterinary medicine, such as:

- Reduced errors resulting from calculations;

- Prescriptions according to guidelines;

- Quick access to patient prescription history;

- Access to accurate new treatment regimens;

- Speeding-up of the chemotherapy administration process and reduced workload;

- Reliable data for the health authorities regarding the number of patients treated, the indications and the necessary amount of drugs;

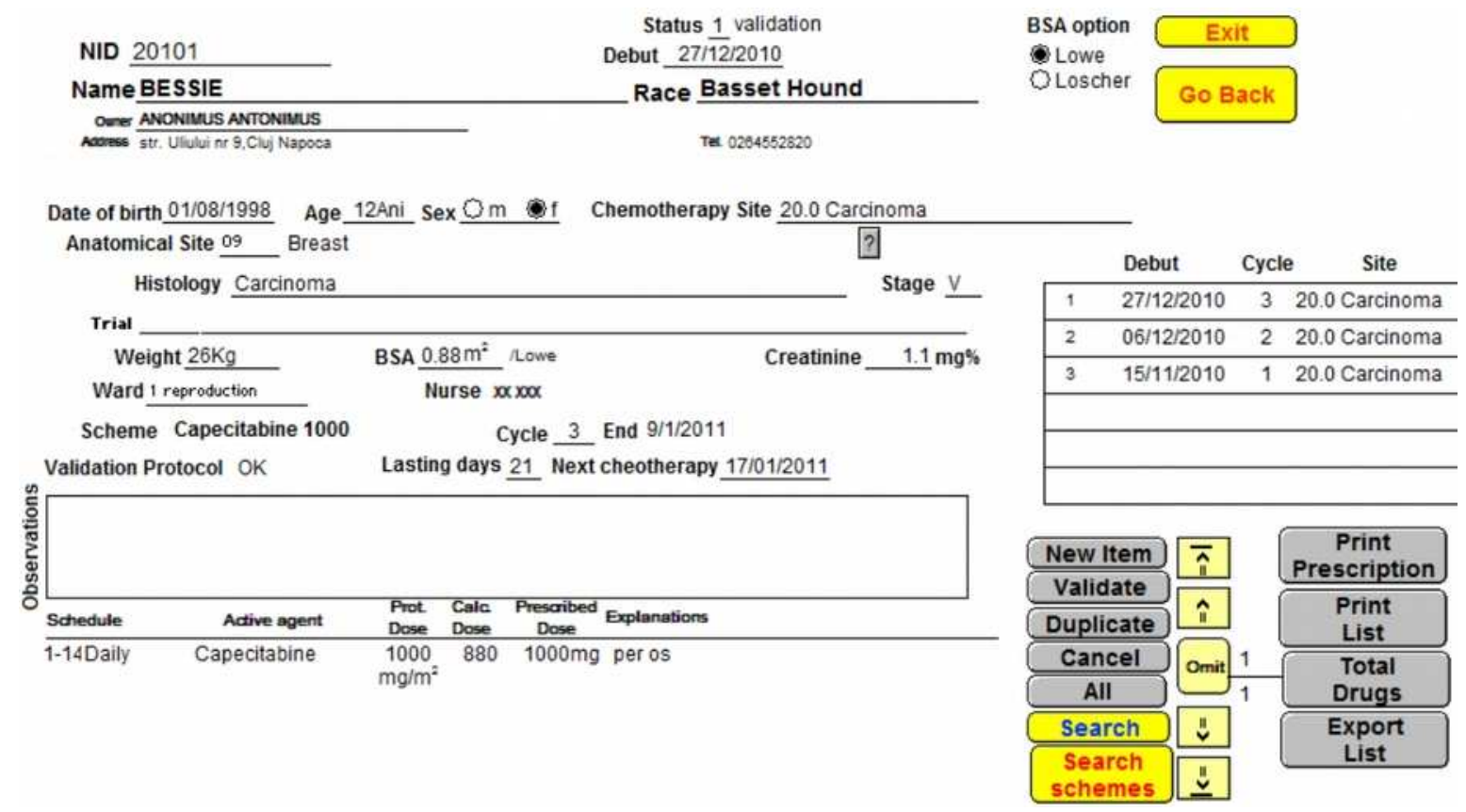

Figure 5. Example of prescription (Canine version). 
- The pharmacy department is supplying cytotoxic drugs having as basis the information from database of prescription.

- The veterinary version of the software helps the creation of a veterinary data base and medical oncology network.

- The browsing system of chemotherapy can be used as an e-learning system.

\section{Acknowledgements}

This paper was published under the frame of European Social Fund, Human Resources Development Operational Programme 20072013, Project no. POSDRU/159/1.5/S/138776.

\section{REFERENCES}

1. CIUlEANU, T. E., N TODOR, Program of Clinical Pharmacography in the Oncology of Solid Tumors (Original title: Program de famacografie clinică în oncologia tumorilor solide), Napoca Star 2014, ISBN: 978-606-690-062-1.

2. http://www.hl7.org/.

3. CERNEA, I. V., A. CHIŞ, N. TODOR, From Passive to Active in the Design of External Radiotherapy Database at Oncology Institute "Prof. Dr. Ion Chiricuță" from Cluj-Napoca, Applied Medical Informatics, vol. 25(3-4), 2009, pp. 7-15.

4. BRICELAND. L. L., T. S. LESAR, D. S. STEIN, Factors Related to Errors in Medication Prescribing, JAMA, vol. 277, 1997, pp. 312-17.

5. http://apps.who.int/classifications/icd10/bro wse/2015/en

6. KUEHR, T., J. THALER, E. WOELL, Chemotherapy Protocols 2013, Current Protocols and "Targeted Therapies". Sandoz, pdl Insbruck 2013, also available on www.chemoprotocols.eu.

7. HERDRICH, K., H. WEINBERGER, Selected Schedules in the Therapy of Malignant Tumors, 17th edition, Part II: Solid Tumors, - Ed. Baxter Oncology $\mathrm{GmbH}$, Sept. 2013.

8. CIULEANU, T. E., L. MIRON, Administration of Therapeutic Agents in Cancer (original title: Administrarea agențílor terapeutici în cancer), Ed. Gr. T. Popa, UMF Iaşi, 2013.

9. DURAND, V., C. LE JEUNNE, Guide Pratique des Medicaments Dorosz, 29-e Edition, Ed. Maloine, Paris 2010.

10. DU BOIS, D., E. F. DU BOIS, A Formula to Estimate the Approximate Surface Area if Height and Weight Be Known, Archives of Internal Medicine, vol. 17(6), 1916, pp. 863-71.

11. GEHAN, E. A., S. L. GEORGE, Cancer Chemotherapy Reports, vol. 54, 1970, pp. 225-235.

12. HAYCOCK, G. B., G. J. SCHWARTZ, D. H. WISOTSKY, Geometric Method for Measuring Body Surface Area: A HeightWeight Formula Validated in Infants, Children and Adults, Journal of Pediatrics, vol. 93, 1978, pp. 62-66.

13. MOSTELLER, R. D., Simplified Calculation of Body-Surface Area, New England Journal of Medicine, vol. 317, 1987, p. 1098.

14. BOYD, E., The Growth of the Surface Area of the Human Body. University of Minnesota, The Institute of Child Welfare, Monograph Series, No. x. London: Oxford University Press, 193.

15. BOXALL, F. E., M. BURNELL, A. H. CALVERT, M. E. GORE, L. A. GUMBRELL, I. R. JUDSON, D. R. NEWELL, S. O'REILLY, Z. H. SIDDIK, E. WILTSHAW, Carboplatin Dosage: Prospective Evaluation of a Simple Formula based on Renal Function, Journal of Clinical Oncology vol. 7(11), Nov. 1989, pp. 1748-56.

16. MAZUMDAR, M., A. SMITH, W. P. TONG, Motzer R.J.: Calvert's Formula for Dosing Carboplatin: Overview and Concerns of Applicability in High-Dose Setting, J. of the National Cancer Institute, vol. 92, September 2000, pp. 1434-1436.

17. BENSADOUN, R. J., P. CANAL, E. CHATELUT, M. C. ETIENNE, E. GUARDIOLA, F. LEGER, N. MAGNÉ, G. MILANO, X. PIVOT, N. RENÉE, Pharmacokinetics of Low-dose Carboplatin and Applicability of a Method of Calculation for Estimating 
Individual Drug Clearance, Annals of Oncology, vol. 14, 2003, pp. 643-647.

18. http://www.eurorec.org/consortium_intern/ seal/sealLevel2.cfm.

19. LOWE, A. D., Alimentary Lymphosarcoma in a 4-year-old Labrador Retriever, The Canadian Veterinary Journal, vol. 45, 2004, pp. 610-612.

20. LOSCHER, W., Plasma Levels of Valproic Acid and Its Metabolites during Continued Treatment in Dogs, Journal of Veterinary Pharmacology and Therapeutics, vol. 4, 1981, pp. 111-119.

21. PAGE, L. R., D. M. VAIL, J. S. WITHROW, Withrow \& MacEwen's Small Animal Clinical Oncology, 5th Edition, Saunders Elsevier, 2013.
22. FRAZIER, D. L., G. S. PRICE, Use of Body Surface Area to Calculate Chemotherapeutic Drug Dose in Dogs. Limitations Imposed by Pharmacokinetic Factors, J. Vet. Intern Med., vol. 12, 1998, pp. 272-278.

23. ADELSON, K. B., M. EVANGELISTA, R. F. HOLCOMBE, Y. C. QIU, P. SPENCERCISEK, C. WHIPPLE, Implementation of Electronic Chemotherapy Ordering: An Opportunity to Improve Evidence-Based Oncology Care, Journal of Oncology Practice, March 2014, pp. e113-e119.

24. http://www.asco.org/practice-research/ehrselection-and-implementation. 